\title{
Striatal and Frontal Pathology: Parkinson's Disease and Patients with Lesions of the Basal Ganglia and Frontal Cortex
}

\author{
Catherine R.G. Jones* and Marjan Jahanshahi**
}

Interval timing is the timing of perceptual or motor events in the millisecond and seconds-range. Everyday examples of perceptual timing are keeping track of elapsed time so that you can estimate that your kettle has boiled, or judging that your favorite television program is due to start. In the laboratory, perceptual timing has been investigated using a wide variety of tasks, including duration discrimination, time estimation, time production and time reproduction. Motor timing is employed in everyday life when we find ourselves clapping in time with music or dancing to the beat. In the laboratory, the more prosaic activity of tapping in time with a regularly paced tone and continuing to do so after cessation of the tone (synchronization-continuation task; S-C) has been used. It has been proposed that at the core of our ability to efficiently time movements and events is a brain-based 'internal clock' that enables precise calculations (see Merchant et al., 2013 for a recent review). However, successful temporal processing requires more than just a working clock. Ancillary cognitive processes are an integral part of a complex neural architecture that gives rise to accurate motor and perceptual timing. As an example, any task in which two intervals are compared requires an on-line representation of the previously presented interval, alongside dynamic updating of the current interval. Therefore, simultaneous maintenance and encoding are required, placing demands on attention and working memory.

This conceptualization of timing is supported by the most dominant model of interval timing, the scalar expectancy theory (SET; Gibbon, 1977; Gibbon et al., 1984). SET conceives that our representations of time are supported in a three-stage process consisting of clock, memory, and decision-making

* School of Psychology, Cardiff University, Uk.

** Sobell Department of Motor Neuroscience and Movement Disorders, UCL Institute of Neurology, London, UK.

(C) CATHERINE R.g. JONES \& MARJAN JAHANSHAHI, 2015 | DOI 10.1163/9789004230699_011 This is an open access chapter distributed under the terms of the Creative Commons 
components (see Figure 10.1). The clock stage is comprised of a pacemaker that emits pulses, with the pacemaker connected to an accumulator. At the onset and offset of an interval that is to be timed, pulses are gated from the pacemaker to the accumulator via a switch, which is operated by a timing signal. The accumulator therefore holds a representation of the current time value, which can be transferred to working memory. The reference memory component provides a more permanent store for important durations (i.e., a standard/ reference duration). The decision process is provided by a comparator, which compares the current time in working memory to the stored time in reference memory. The 'scalar' part of the model comes from applying the observation that human (and animal) timing conforms to the scalar property, whereby the standard deviation of timed responses increases with the mean of the interval being timed. There are challenges to the SET model of interval timing, particularly regarding the biological plausibility of the model (e.g., Matell and Meck, 2000, 2004). However, the model is a salient demonstration of the widespread belief that clock processes are supported by a range of cognitive operations

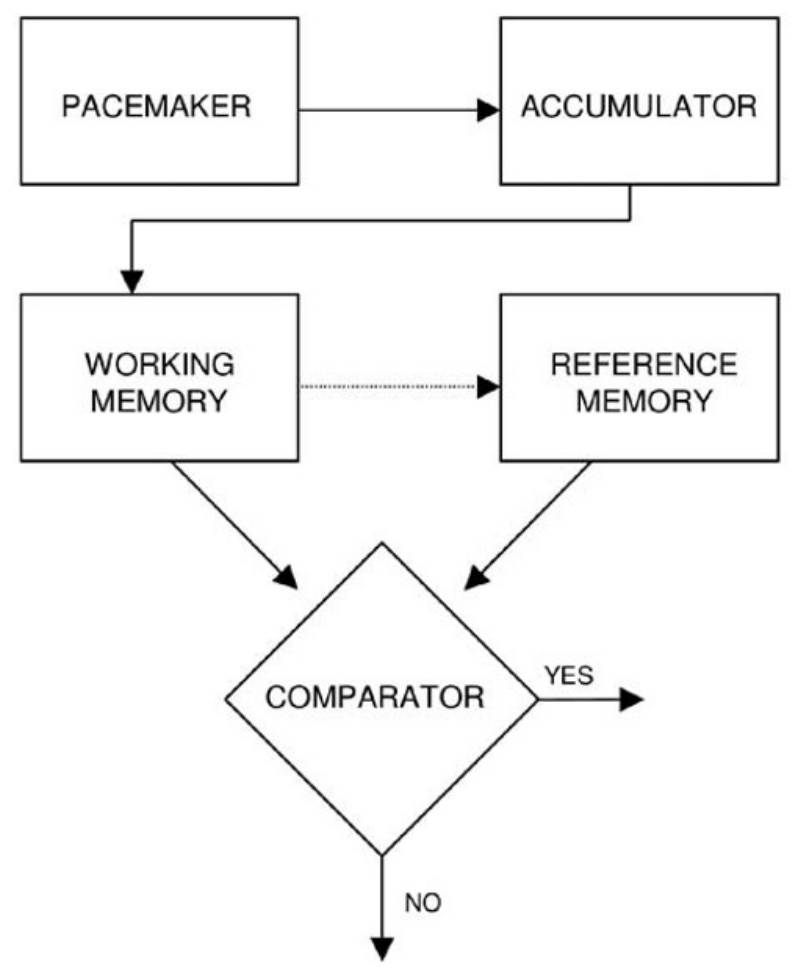

CLOCK
PROCESSES

MEMORY PROCESSES

FIGURE 10.1 Scalar expectancy theory (Gibbon, 1977; Gibbon et al., 1984). 
(attention, memory, decision making), which may have their own distinct neural correlates.

The focus of clinical investigation into interval timing has been on identifying the structure(s) that are integral to the clock process. A large body of literature has investigated the role of the basal ganglia, particularly the temporal profile of individuals with Parkinson's disease (PD). PD is the result of a loss of dopamine producing neurons in the substantia nigra pars compacta (SNc), a pathology that leads to akinesia (lack and poverty of movement), bradykinesia (slowed movement initiation and execution), rigidity (increased muscle tone), and tremor at rest. However, aside from the cardinal motor difficulties, cognitive and affective difficulties are also prevalent. These include depression, anxiety, apathy, hallucinations and delusions, sleep disturbance and cognitive decline (e.g., Chaudhuri and Schapira, 2009). Particularly, impaired executive function is observed from early on in the disease (for a review see Dirnberger and Jahanshahi, 2013), with more widespread cognitive decline emerging as the disease progresses. Executive functions are primarily mediated by the prefrontal cortex and include a range of cognitive processes, including planning, task switching, and inhibition. These are integrated and coordinated to enable achievement of goal-oriented cognitive operations, particularly when complex or novel. Occurrence of executive dysfunction in PD is not surprising, given that the basal ganglia have multiple connections to the frontal cortex, in the form of a series of frontostriatal loops (Alexander et al., 1986). The pathophysiology of PD is complex and changes in the course of the illness. The motor frontostriatal loop, connecting the putamen with the supplementary motor area (SMA), is the site of the primary dysfunction in PD, but other frontostriatal circuits become affected as the disease progresses. These include the associative circuit between the caudate and the dorsolateral prefrontal cortex (DLPFC), as well as the limbic circuit between the ventral striatum and the anterior cingulate. The frontal cortex, particularly the DLPFC, has an established role in a range of cognitive processes, such as working memory and decision making (e.g., D'Esposito, 2007), which SET proposes are necessary for efficient interval timing. Therefore, involvement of additional circuits in PD means that it can be difficult to tease apart whether the timing difficulties are being driven by a core timing dysfunction, or by disruption of cognitive processes.

One way of exploring the differential contributions of the basal ganglia and frontal cortex to motor and perceptual timing is to investigate the timing profile of patients with circumscribed damage to the basal ganglia or frontal cortex. This includes individuals with traumatic brain injury, stroke or tumors. The capacity of the human brain for the development of compensatory neural 
circuits means that caution must be exercised when investigating the effects of acquired brain injury. Further, studies are limited by the difficulty of finding patients with comparable pathology, as well as with lesions that are solely limited to the region of interest. However, such patients provide an unparalleled insight into the function of the basal ganglia and frontal cortex in temporal processing. Therefore, the present chapter draws on evidence from patients with circumscribed lesions and patients with PD to better understand the roles of the basal ganglia and frontal cortex in interval timing. There is a great deal of heterogeneity among patients with acquired lesions. We have limited our evaluation to those with a discrete lesion, e.g., caused by tumor, stroke or head injury. Patients with degenerative disease (e.g., dementia) have not been included. For the frontal cortex, studies that investigate a range of lesions sites have been included if the reporting of the data enables conclusions to be drawn about the specific contribution of the frontal cortex. In complement to the current chapter, Chapter 8 provides an overview of timing in neurodegenerative disorders of the basal ganglia, particularly PD and Huntington's disease. Chapter 11 also includes a summary of functional imaging research in PD, which is not covered in the current chapter. We start with an overview of the tasks that have been employed to assess timing, before reviewing the empirical evidence.

\section{Measures of Perceptual and Motor Timing}

Perceptual timing tasks are far more varied than motor timing tasks, and refer to any subjective judgment of time that is not defined by movement. Sometimes a motor response in a perceptual task may be tied to the temporal decision (e.g., pressing a button when a certain period of time is perceived to have elapsed), thus the categorization of tasks is not clear cut. Indeed, studies have shown a significant correlation between performance on motor and perceptual timing tasks (e.g., Keele et al., 1985; Merchant et al., 2008), which is compatible with there being a shared neural substrate. Further, although this chapter focuses on the perceptual vs. motor distinction there are other ways of categorizing temporal tasks, including the length of interval used (e.g., Lewis and Miall, 2003) and whether timing is an explicit demand of the task or implicit and emergent (e.g., intercepting a moving target; see Coull and Nobre, 2008).

Perceptual timing is commonly measured in the visual or auditory modality, using presentation of simple auditory (e.g., pure tone) or visual (e.g., small square) stimuli to define temporal intervals. Another variation is whether the intervals are be 'filled' (i.e., the stimulus is present for the duration of the interval) or 'unfilled' (i.e., the onset and offset of the interval is bounded by two 
stimuli, but the actual interval is empty). Filled intervals can sometimes take the form of an active and additional task, for example, counting or reading aloud random numbers. All perceptual tasks share certain cognitive demands including sustained attention to the temporal interval(s) as well as decisionmaking/evaluative processes. However, the reliance on other cognitive processes varies with task demands. The duration discrimination task presents two stimuli sequentially and requires the participant to discriminate which is longer, or whether the second stimuli is longer or shorter than the first. The appeal of this task is that the perceptual temporal decision is not confounded by movement. At the cognitive level, the demand on working memory is high as both intervals need to be simultaneously held on-line and compared.

The time estimation task and time production task share similarities, as both require units of time to be accurately applied to an interval. In the time estimation task the participant has to estimate the length of a presented interval to the nearest second. In contrast, the time production task requires the participant to indicate (e.g., press a button) when a specified interval of time has elapsed. Therefore, in both of these tasks the participant has to map their understanding of the common units of time onto their internal sense of time passing. The time reproduction task presents an interval of time but rather than compare it to another interval, the participant has to reproduce the duration of the interval as accurately as possible using a response key. Similar to the duration discrimination task, this places considerable demand on working memory. For the time estimation, production, and reproduction tasks, sometimes participants are instructed to count out intervals, either at a self-paced and self-preferred rate, at a self-paced but specified rate (e.g., 1 s), or at an externally paced rate (e.g., provided by a metronome). These modifications mean that participants are both required to accurately perceive a discrete interval and time a short continuous sequence that is intrinsically tied to motor production. Thus, many purported perceptual timing tasks have an implicit motor timing element. Chronometric counting is distinct from interval timing, albeit with possible overlap (e.g., Hinton et al., 2004). Therefore, some studies require that random numbers are read aloud to inhibit counting. However, all of these tasks are ostensibly dual task paradigms, which is differentially demanding for patients with neurological damage compared to healthy controls (e.g., Brown and Marsden, 1991; Perbal et al., 2005). Although requiring participants not to count or read random numbers may present as an obvious solution, it then becomes difficult to control the mental activities that participants engage in during the task.

Three classic tasks from the animal timing literature, the peak interval procedure, the temporal generalization task and the temporal bisection task have 
been adapted to investigate perceptual timing in patient studies. These three tasks share a commonality in demanding that a learnt interval be stored in short term memory and recalled when required. In the peak interval procedure, the participants are trained on a visually presented target duration that they will need to reproduce. In the testing phase, the visual stimulus is presented for a longer period. Participants have to press a button when they think the target duration has elapsed but they are instructed to make multiple guesses; pressing the button before the estimated duration has elapsed and continuing until they judge it has passed. In many ways analogous to a time reproduction task, this procedure enables a response curve to be plotted, showing a peak at the time where responses are most frequent. With time plotted on the x-axis, a curve with a peak shifted to the right indicates relative overestimation, whereas a peak shifted to the left indicates underestimation. In the temporal generalization task participants are repeatedly exposed to a target duration, which becomes learnt. During the testing phase participants have to indicate whether the durations they are presented with are the same or different to the learnt target. The proportion of 'yes' responses can be plotted to create a temporal generalization gradient, illustrating the probability of a response as a function of signal duration. With duration plotted on the $\mathrm{x}$-axis, a rightward shift in the peak of the generalization function would indicate overestimation of the target duration, whereas a leftward shift would suggest underestimation. The temporal bisection task requires participants to learn a 'short' and a 'long' target duration. In the testing phase, participants are presented with a range of durations including the learnt targets. Each duration has to be classified as more similar to the short or long target. The resultant sigmoid curve plots the probability of making a 'long' response as a function of stimulus duration. With durations plotted on the x-axis, a leftward shift in the curve indicates a relative overestimation of time. The bisection point (or point of subjective equality; PSE) is the duration at which both responses occur with equal probability.

Motor timing is assessed using a repetitive tapping task, often called the S-C task. First, the participant taps in time with a regularly paced cue, typically an auditory pure tone. Following a criterion number of taps the pacing cue is switched off and the participant has to maintain the same rhythm unaided. Thus, there are two discrete phases to the task, a synchronization phase and a continuation phase, which are measured separately. The accuracy of the tapping rate, usually measured by the mean inter-response interval, is important in determining whether tapping is unusually slow or fast. Variability has classically been measured using the Wing and Kristofferson (1973a,b) model, which decomposes tapping variability into 'clock' and 'motor' components, although 
the model is not without caveats (see Jones et al., 2011). The S-C task is considered to be more 'automatic' and less cognitively demanding than perceptual tasks, particularly when short inter-tap intervals are used (Lewis and Miall, 2003).

\subsection{Perceptual Timing}

Perceptual timing tasks in patients with PD are summarized in Table 10.1. Of the 37 tasks, there are significant differences between the PD and control group on 23 tasks $(62 \%)$. Data have indicated underestimation on time estimation tasks (Lange et al., 1995; Pastor et al., 1992a) and overestimation on time production tasks (Lange et al., 1995; Jones et al., 2008) in patients with PD. This pattern of results is compatible with a slowed internal clock (although see Wojtecki et al., 2011). The slowed clock hypothesis is particularly compelling as it neatly reflects the bradykinetic presentation of patients with PD. However, many other studies fail to find evidence of impairment on these two tasks (Perbal et al., 2005; Riesen and Schnider, 2001; Wearden et al., 2008; Wild-Wall et al., 2008). Further, patterns of impairment on other timing tasks are not amenable to the slowed clock hypothesis. For example, in the time reproduction task the internal clock is being used to both estimate and reproduce; this means the speed of the clock could not influence the findings, assuming the rate was regular.

The time reproduction task is the most discriminating task, and two thirds of studies reported some degree of impairment in PD (see Table 10.1). It is conceivable that the motor response, which is intrinsically tied to the temporal decision, may compound or drive the poor performance on the task. However, this interpretation does not explain the pattern of within-task overestimation on relatively short intervals compared to underestimation on relatively long intervals (see Koch et al., 2004; 2005). Patients with PD have also shown evidence of impairment on the duration discrimination task, although preserved performance is also reported (see Table 10.1). Harrington et al. (1998a) reported dissociation in PD between impaired duration discrimination and preserved frequency discrimination, which is compatible with a timing-specific deficit and not general cognitive dysfunction. However, the duration task, unlike the frequency task, requires attention be maintained for the duration of the presented stimuli. Therefore, the additional attentional demands of the duration task provide an alternative explanation for the pattern of results. 


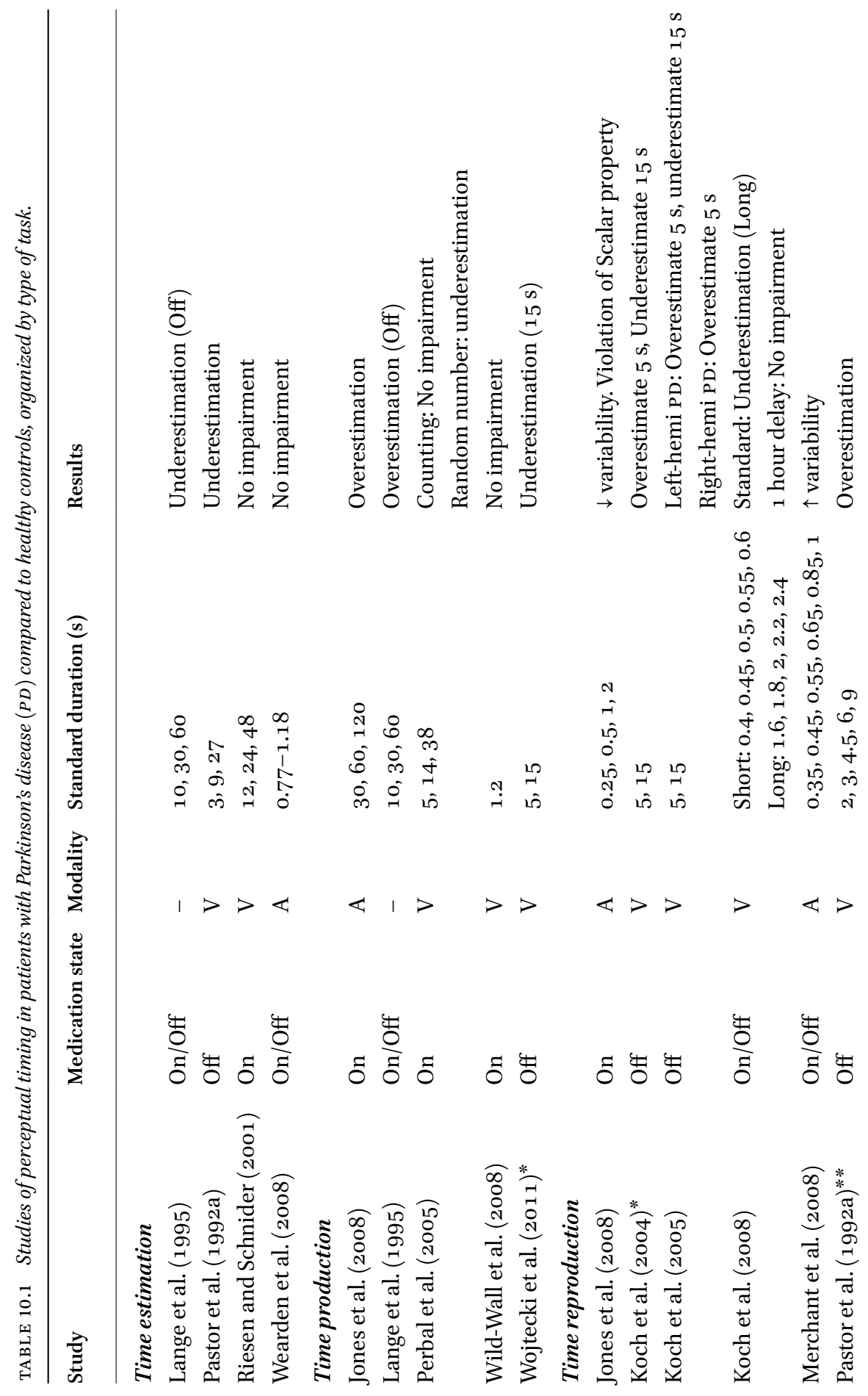




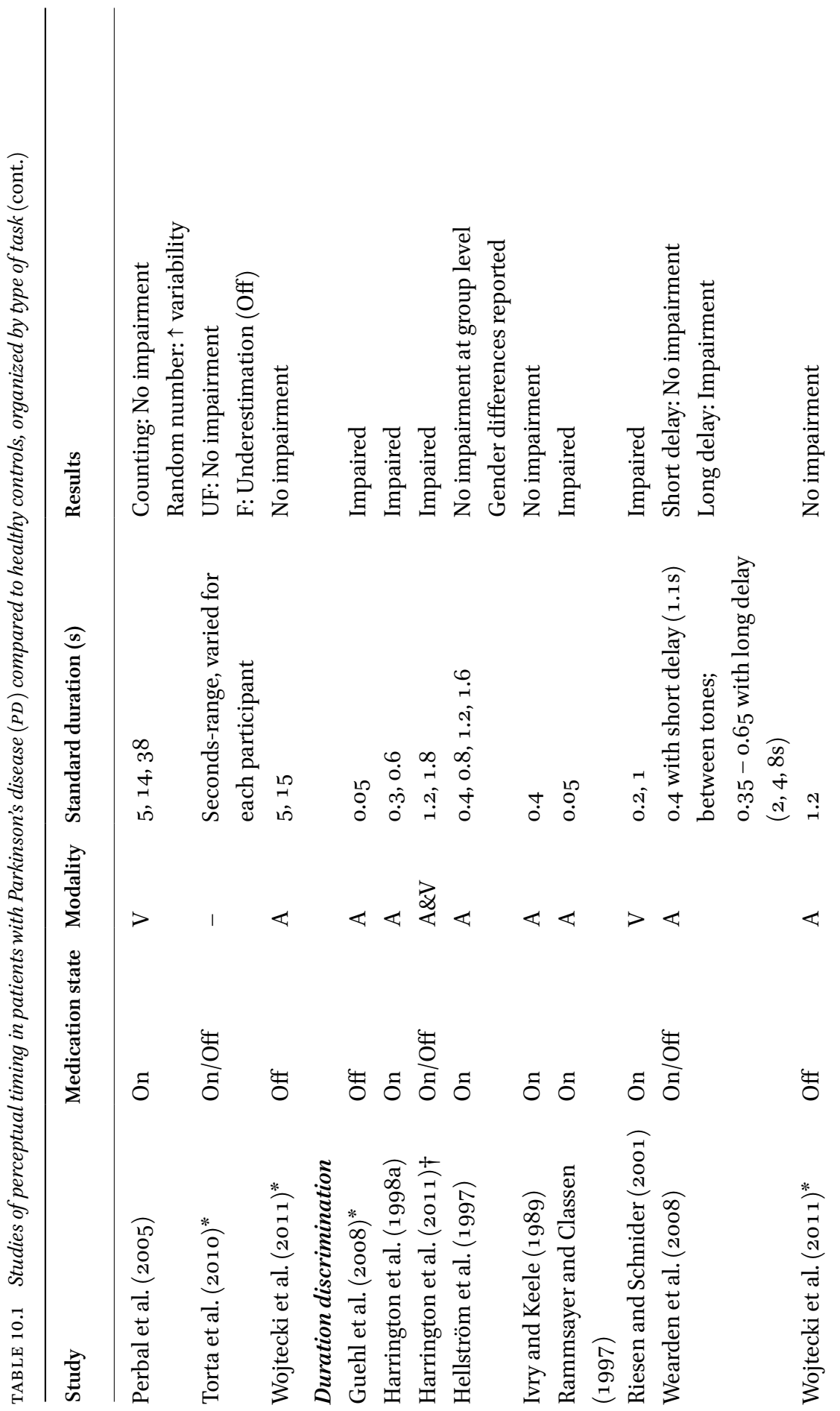




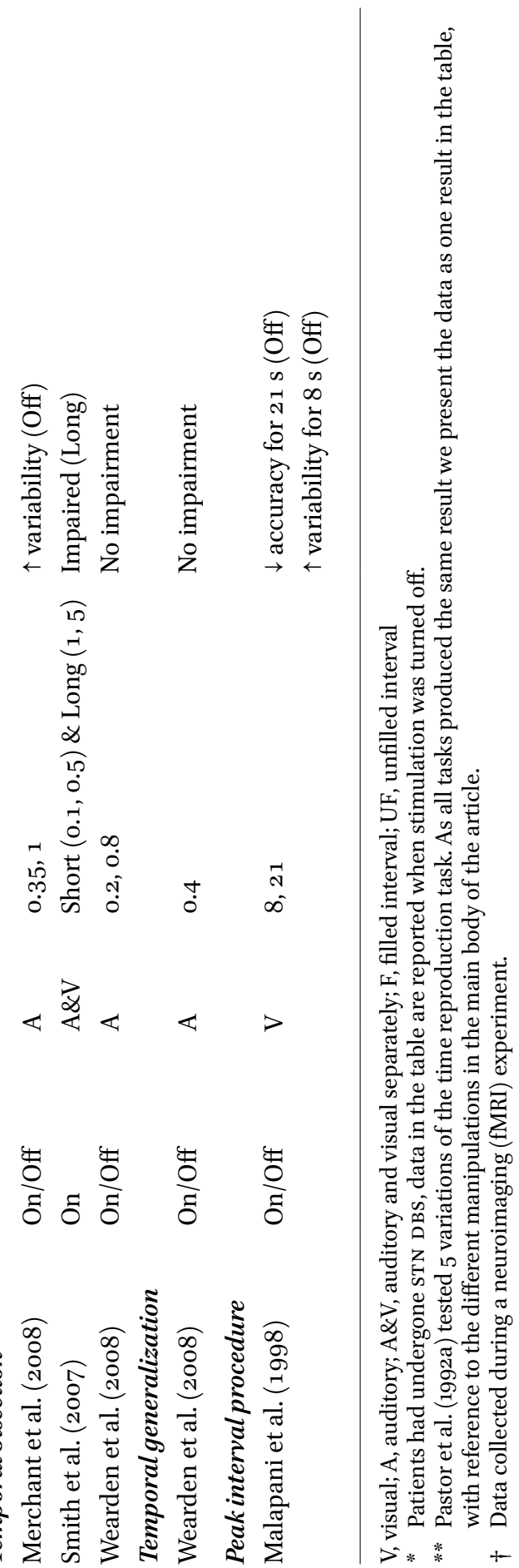


The temporal generalization and bisection tasks present a mixed picture, with no consistent evidence of impairment (see Table 10.1). However, the peakinterval procedure has produced more convincing results. Here patients show evidence of a 'migration' effect whereby relatively short intervals are overestimated and relatively long intervals are underestimated (Malapani et al., 1998; Malapani et al., 2002). As previously alluded to, this effect has been replicated in studies of time reproduction (Koch et al., 2004; 2005). Systematic investigation of the pattern of responding under different conditions has indicated that deficits in the storage and retrieval of the durations in memory contribute to the migration effect (Malapani et al., 2002). Further, the effect is abolished if the reproductions of the short and long intervals are separated by a delay of one hour (Koch et al., 2008). The profile of performance across tasks investigating the migration effect are, therefore, compatible with a cognitive explanation for the perceptual timing difficulties seen in PD.

Other studies also present data that are compatible with a cognitive explanation. For example, Guehl et al. (2008) employed a duration discrimination task where the standard interval of 5oms was defined by two clicks. Although participants with PD were impaired on this task they were unimpaired on a version where trains of clicks paced at 5oms intervals were used and participants had to decide which of two trains had one long interval (>5oms) in the middle. Therefore, although the durations being judged were identical across the two tasks, the task context was different. The authors suggested that performance is impaired on the standard duration discrimination task because the onset of the stimuli cannot be predicted as easily i.e., it requires a greater allocation of attentional resources. An attentional explanation was also put forward by Torta et al. (2010), who found that participants with PD were impaired when reproducing the amount of time it had taken them to complete a simple motor task but unimpaired when the interval to reproduce was unfilled. The dissociation was argued to be driven by the demands of the concurrent motor task, which left less attentional resources for the timing task. Finally, Wearden et al. (2008) assessed patients with PD on five different perceptual timing tasks. The only task that they were impaired on was a duration discrimination task that required the standard interval (350-65oms) to be held in memory for $2-8 \mathrm{~s}$. Therefore, impaired memory function may also be a contributing factor to performance.

As was mentioned in the Introduction of this chapter, PD is principally a movement disorder but cognitive deficits are a feature of the disease. These include executive dysfunction and mild cognitive impairment in the early stages, and dementia in the later stages (Dirnberger and Jahanshahi, 2013; Kehagia, Barker, and Robbins, 2013). If cognitive difficulties contribute to the 
temporal profile seen in PD then tasks with additional cognitive load should be more likely to elicit impairment. In support of this, the majority ( 5 of $6 ; 83 \%$ ) of tasks that required random numbers to be read out during timing reported a deficit (Koch et al., 2004, 2005; Malapani et al., 1998; Riesen and Schnider, 2001). A cognitive explanation would also predict greater impairment at longer intervals, where demands on memory and attention would be more extensive. Indeed, Jones et al. (2008) used exploratory factor analysis to establish that the time production of seconds-range intervals and a measure of attention (Paced Auditory Serial Addition Test) shared common variance and were distinct from time reproduction and warned reaction time tasks involving millisecondrange interval timing. If the tasks in Table 10.1 are divided by the arbitrary cutoff of $1 \mathrm{~s}$ (i.e., standard intervals of $1-1000 \mathrm{~ms}$ vs. standard intervals $>1000 \mathrm{~ms}$ ), 6 of $15(40 \%)$ studies using the shorter intervals reported poor performance in the PD group, compared to 16 of $26(62 \%)$ using the longer interval range. The proportion of those performing poorly at longer intervals is increased further if the cutoff is set at $5 \mathrm{~s}(71 \% ; 12$ of 17$)$. This pattern across studies indicates that longer intervals are more challenging for patients with PD. However, the presence of deficits in tasks with very short intervals (e.g., Guehl et al., 2008; Rammsayer and Classen, 1997) as well as preserved performance in studies where the intervals are substantial (e.g., Perbal et al., 2005; Riesen and Schnider, 2001; Wojtecki et al., 2011) needs to be considered. Further, neuroimaging research has found basal ganglia activation in both millisecond and secondsrange temporal processing (Jahanshahi et al., 2006).

Teasing apart the specific factors that contribute to temporal dysfunction in PD can be aided by investigating correlates of poor performance. Pastor et al. (1992a) found that time reproduction was associated with measures of natural tapping rate, reaction time, and movement time. This suggests that perceptual timing deficits relate specifically to bradykinesia in PD. However, although some studies have found correlations between perceptual timing and disease severity (Pastor et al., 1992a; Wearden et al., 2008) this association is not always found (Rammsayer and Classen, 1997; Smith et al., 2007). In terms of temporal deficits being driven by non-motor deficits, Rammsayer and Classen (1997) reported a significant correlation in PD between increased self-reported depression and greater impairment on a duration discrimination task. Further, poor performance on the time production task (Wild-Wall et al., 2008) and time reproduction task (Perbal et al., 2005) was associated with higher levels of cognitive decline. In contrast, Merchant et al. (2008) found that performance on a range of cognitive tasks (working memory, go/no-go reaction time, and verbal learning) did not discriminate those with PD who performed well or poorly on a range of timing tasks. However, their timing tasks all used intervals 
$\leq 1 \mathrm{~s}$, and it may be that general cognitive ability is an additional source of variance only for the more cognitively demanding seconds-range intervals.

\subsection{Motor Timing}

Motor timing studies in PD are summarized in Table 10.2. The influential Wing and Kristofferson $(1973 \mathrm{a}, \mathrm{b})$ model was designed to characterize tapping in the continuation phase. As such, the synchronization phase has not been analyzed in most studies. However, both healthy participants and those with PD perform better at synchronization than continuation tapping (e.g., Jones et al., 2011) and there is no convincing evidence that the pattern of impairment in PD differs significantly between the two phases (e.g., Jones et al., 2011; Joundi et al., 2012; Pastor et al., 1992b; Wojtecki et al., 2011).

Given that PD is a movement disorder it is perhaps not surprising that motor timing deficits are reported more consistently than perceptual timing deficits. Of the 13 studies reported in Table 10.2, 10 (77\%) report group differences in variability or accuracy. However, closer inspection reveals a more complex picture. For accuracy, tapping rate on the S-C task in PD has been shown to be faster (Harrington et al., 1998a; Ivry and Keele, 1989; Jones et al., 2011; O'Boyle et al., 1996), slower (Pastor et al., 1992b), and unimpaired (Duchek, Balota, and Ferraro, 1994; Joundi et al., 2012; Spencer and Ivry, 2005; Wojtecki et al., 2011). One interpretation for these inconsistent findings is that performance depends on the duration being timed. It has been proposed that individuals with PD can demonstrate 'hastening' and festination at the shorter intervals (Claassen et al., 2013; Jones et al., 2011). Festination is a clinical phenomenon often observed in PD and is the tendency to speed up when performing a repetitive movement. A review of the motor timing literature (see Jones et al., 2011; Jones and Jahanshahi, 2014) indicates that the interval range of 400-6ooms is the threshold at which performance switches from impaired to unimpaired in PD. In contrast, there does not seem to be a threshold effect for variability, although having tested patients on a range of intervals $(250,500,1000$, and 200oms), Jones et al. (2011) found that variability for both patients with PD and healthy controls was lowest at 50oms.

As the clinical motor features of PD are compatible with difficulties in motor timing, it is surprising when studies fail to find an association between clinically assessed motor dysfunction and performance on the S-C task (O'Boyle et al., 1996; Spencer and Ivry, 2005). However, positive associations have been reported by some (Harrington et al., 1998a). To our knowledge, there are no studies that have assessed whether there are cognitive or affective correlates of motor timing performance in PD, which should be addressed in future research. 


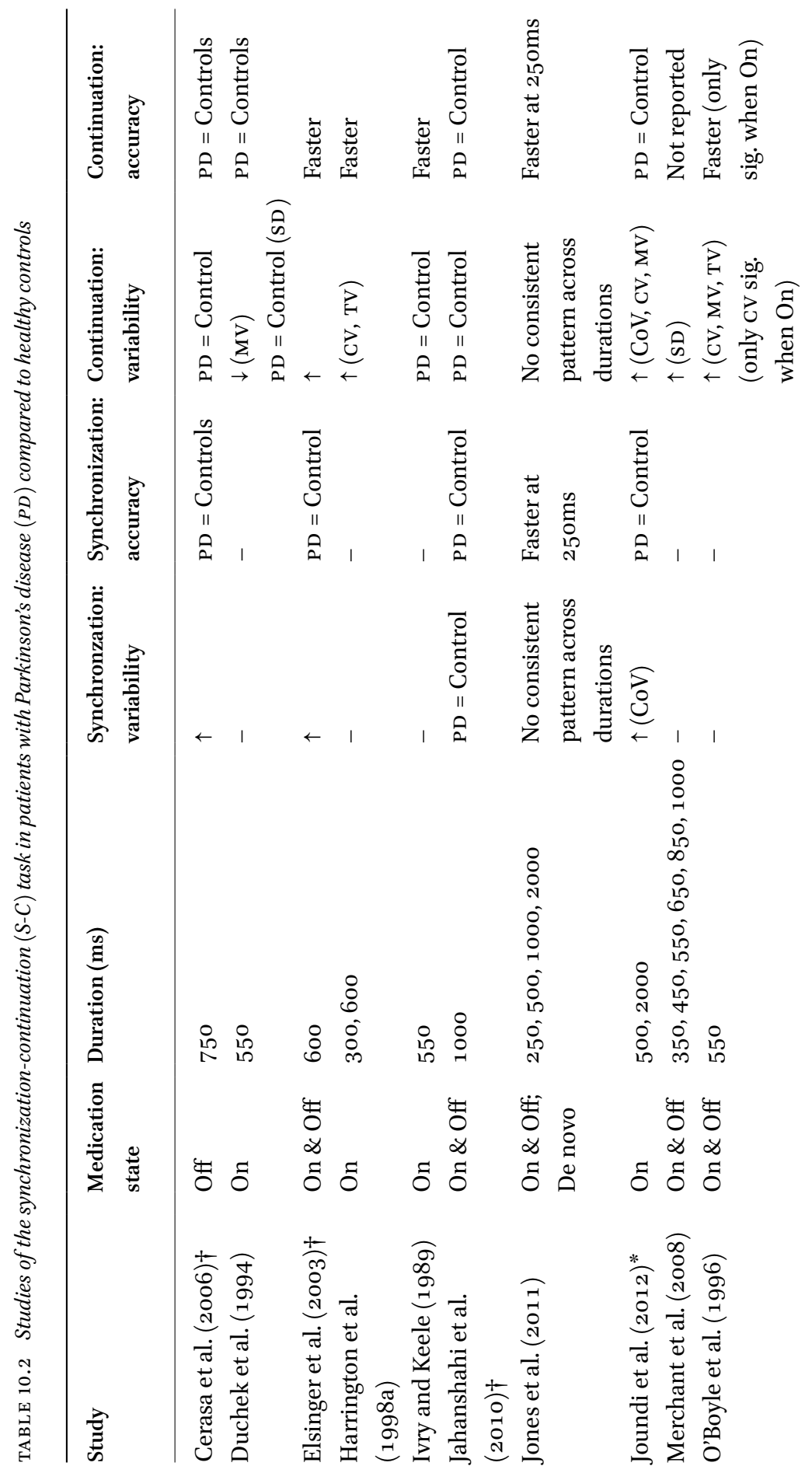




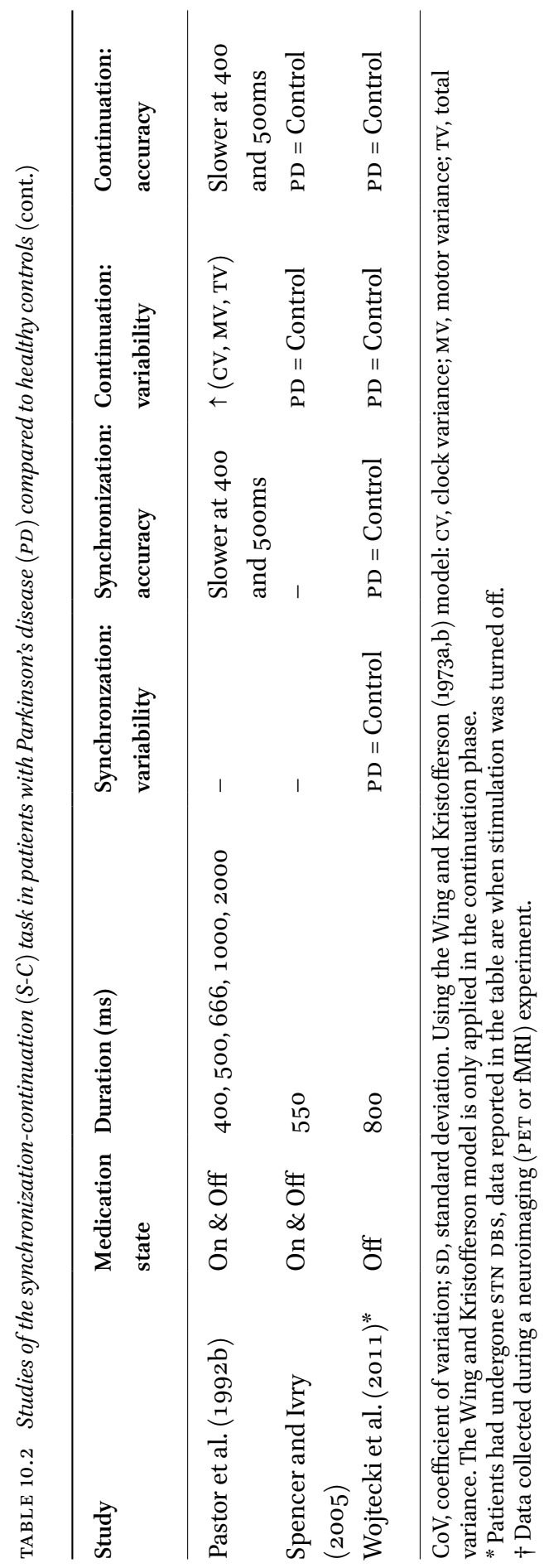




\subsection{Summary}

There is clear evidence of impairment in both motor and perceptual timing in the milliseconds and seconds-range in PD, although also a considerable number of studies do not report deficits. Dopaminergic medication can benefit timing performance in $\mathrm{PD}$. This was the case for $57 \%$ (8 of 14) of the perceptual timing tasks (see Table 10.1) and $40 \%$ ( 2 of 5 ) of the motor timing tasks (see Table 10.2) that reported impairment 'off' medication. Interpreting studies that do not report a positive effect of medication is difficult given issues such as individual differences in response to medication, the varying types of medication used, and study-specific differences such as the length of medication withdrawal. Historically, perceptual timing dysfunction has been interpreted as evidence of a slowed internal clock. However, the pattern of deficits across tasks is often not compatible with this explanation. Further, evidence suggests that compromised frontal activity in PD, likely affecting ancillary cognitive processes, may be contributing to or driving the difficulties in perceptual timing. Of course, it is possible that poor temporal processing in $\mathrm{PD}$ is driven by a combination of a core timing deficit and cognitive impairment. One piece of evidence that provides a compelling argument for a core timing deficit is work by Grahn and Brett (2009). Here a rhythm discrimination task established that patients with PD were as proficient as controls at discrimination when the rhythm did not contain a beat but showed poorer performance when a beat was present. Rhythms with a beat structure are easier to discriminate than rhythms without a beat, so a global cognitive or perceptual impairment could not be driving the timing deficit. What is clear is that the data from studies of PD support the hypothesis that the integrity of the basal ganglia and frontal cortex are essential to motor and perceptual timing.

Patients with focal lesions to the basal ganglia provide an important approach for assessing the contribution of the basal ganglia to temporal processing. The primary difference between patients with basal ganglia lesions and patients with PD is that the latter have a degenerative disorder that involves areas and systems of the brain beyond the basal ganglia. Therefore, patients with acquired lesions to the basal ganglia allow a more constrained interpretation of the contribution of the basal ganglia to timing function. Ultimately, these data enable us to consider whether the timing dysfunction observed in PD is driven by top-down cognitive factors rather than a critical impairment to an internal timekeeper. 
Aparicio et al. (2005) assessed 6 patients with right-sided lesions of the basal ganglia and found no evidence of impairment on the S-C task with an inter-tap interval of 40oms. A similar study was conducted more recently by Schwartze et al. (2011) who adopted an innovative adaptive version of the S-C task. Here, 10 patients with unilateral lesions of the basal ganglia (8 left- and 2 right-sided) were assessed tapping at their most comfortable rate and on an adaptive S-C task, which included a change in tempo (acceleration or deceleration over 3 intervals) at the end of the synchronization phase. Compared to healthy controls, the patient group showed more variable tapping when required to tap at their most comfortable rate and showed a more heterogeneous distribution of individual rates. This suggests difficulty with internally generated temporal pacing following basal ganglia damage, perhaps not surprising given that a primary projection site of the basal ganglia, the pre-supplementary motor area, has a critical role in initiating freely chosen movement (e.g., Halsband et al., 1993). For the S-C task, there was no fundamental difference in tapping accuracy between groups, and differences in variability were significant in a $t$-test but not in a more comprehensive ANOVA. These findings are therefore superficially compatible with Aparicio and colleagues. However, Schwartze et al.'s innovative adaptive paradigm uncovered a novel finding. They found that patients were less able to correct their tapping rate to the accelerated or decelerated rhythm. Analysis of the profile of error correction revealed that the patients were having difficulty with period, rather than phase, correction. Period correction is thought to reflect an internal timekeeper and is assumed to rely on cognitive control. As such, the authors speculate that impairment to attention-dependent aspects of temporal processing lead to deterioration in performance in individuals with basal ganglia lesions when the rhythm changes. Reflecting this interpretation, the motoric disturbance was accompanied by reduced sensitivity for accurately identifying whether the tempo had changed, and in which direction.

The previous studies have only used patients with unilateral lesions, which do not necessarily lead to the expression of behavioural dysfunction. Indeed, when required to tap as fast as possible, Aparicio et al.'s (2005) patients did not show significantly reduced tapping speed. Whether the absence of clear behavioural markers is due to compensation by the unaffected hemisphere or reflects a relatively minor insult is unclear. Regardless, it makes interpreting intact temporal performance difficult. Coslett et al. (2010) addressed this issue by assessing two patients with bilateral damage to the basal ganglia on a comprehensive battery of perceptual and motor timing tasks. Importantly, both patients showed significant evidence of motor-related dysfunction, including dystonic posturing and akinesia; whereas their cognitive profiles did not indicate any areas of impairment. In contrast to the data presented by Schwartze 
et al. (2011), both patients performed well on measures of perceptual timing. These included time estimation, reproduction, and production tasks in the seconds-range (2-12 s) and a duration discrimination task of both millisecond (300, 60oms) and seconds-range $(2,8 \mathrm{~s})$ intervals. However, when required to perform the S-C task with an inter-stimulus interval of 40oms, one patient showed abnormally short responses, while the other showed significantly elevated variability. This finding appears in contrast to Aparicio et al. and Schwartze et al., neither of whom found evidence of impairment with standard S-C tapping. However, the participants in Schwartze et al.'s study tapped with their least affected (i.e., ipsilesional) hand. Aparicio et al.'s participants were assessed on both hands and the pattern of data indicated aspects of performance, particularly motor variability, were more compromised in the contralesional hand. However, this effect did not reach statistical significance.

The pattern of data across studies highlights the difficulty in separating the non-specific aspects of motor preparation and execution from the specific temporal contribution. Schwartze et al.'s approach of manipulating the temporal component of the task whilst the motor demands remain constant is an elegant way of dissociating the processes that contribute to motor timing. Schwartze et al.'s patients demonstrate a difficulty in attending to time, which is a deficit that should have compromised performance on Coslett et al.'s perceptual timing tasks, particularly as most were in the seconds-range. However, Schwartze et al.'s S-C task required the monitoring of rhythm changes, introducing a perceptual timing element to a motor timing task that is typically considered to be processed automatically and with limited cognitive engagement (Lewis and Miall, 2003). This 'double hit' may have created a demanding task that was better placed to elicit subtle difficulties in attending to time.

In conclusion, the majority of the motor timing data do not support a link between basal ganglia pathology and motor timing dysfunction. However, the finding (Coslett et al., 2010) of motor timing impairment in patients with bilateral lesions, in contrast to patients with unilateral lesions, suggests that the basal ganglia are important for motor timing but that both hemispheres need to be compromised. Pervasive cognitive deficits are absent in patients with lesions to the basal ganglia, in contrast to individuals with $\mathrm{PD}$, which provides greater clarity when interpreting results. Impairment in the detection and response to rhythm change (Schwartze et al., 2011) is compatible with the monitoring of time being compromised and merits further investigation. However, Coslett et al.'s (2010) multi-task assessment of perceptual timing found no evidence of impairment in their two patients with bilateral lesions. Clearly, there is not enough data on perceptual timing in individual with basal ganglia lesions to draw any firm conclusions and further research is needed. 


\subsection{Perceptual Timing}

Studies reporting perceptual timing in patients with lesions to the frontal cortex are summarized in Table 10.3. As with patients with basal ganglia lesions, these patients allow a more specific investigation of the contribution of a particular brain region than is afforded by studying patients with a degenerative disorder. The clearest finding from surveying the data in Table 10.3 is that perceptual timing impairments in frontal patients are more common than in patients with PD and are present in 88\% (14 of 16 ) of tasks across 11 studies. Notably, these impairments are seen for both millisecond and seconds-range intervals. Taking a closer look at the specific pattern of findings is important for understanding the nature of the deficit(s) that are leading to the profile of impairment.

The frontal cortex performs a wide range of cognitive operations and the locus of the lesion site is an important consideration for interpretation. The orbitofrontal (OFC) is associated with impulsive behavior (e.g., Torregrossa et al., 2008), which is intrinsically linked to insensitivity to reward and punishment (Berlin et al., 2004). Delay aversion is a critical component of impulsivity and may impact on any task where a response has to be withheld until a specific time. In keeping with this, Berlin et al. found that patients with lesions to the ofC underestimated the second-range intervals that they were required to produce. They also overestimated the duration of seconds-range intervals that were presented to them. The increased time estimation and decreased time production were positively correlated with the 'attention' subscale of the Barrett Impulsiveness Scale, a self-report impulsivity questionnaire. Whether lesions to the OFC cause a speeded internal clock, which contributes to impulsive behaviour (i.e., the subjective experience of time passing slows, which exacerbates intolerance to reward delay), or if the impulsivity itself drives the temporal distortion (e.g., difficulty in waiting for the interval to end makes it seem longer) is an important question. Of course, as Berlin et al. discuss, it is also possible that OFC patients have a speeded clock and an intolerance of delayed gratification. In contrast to those with ofC lesions, patients with damage to the prefrontal cortex (PFC), including the DLPFC, did not show atypical timing behaviour. This is perhaps surprising given the role of the DLPFC in attention (e.g., Katsuki and Constantinidis, 2012).

Well-reported case studies can give a unique insight into the qualitative temporal distortions experienced by those with focal lesions. Binkofski and Block (1996) detail a patient with a tumor in the left superior PFC, including the DLPFC, who described experiencing time as passing more quickly than 


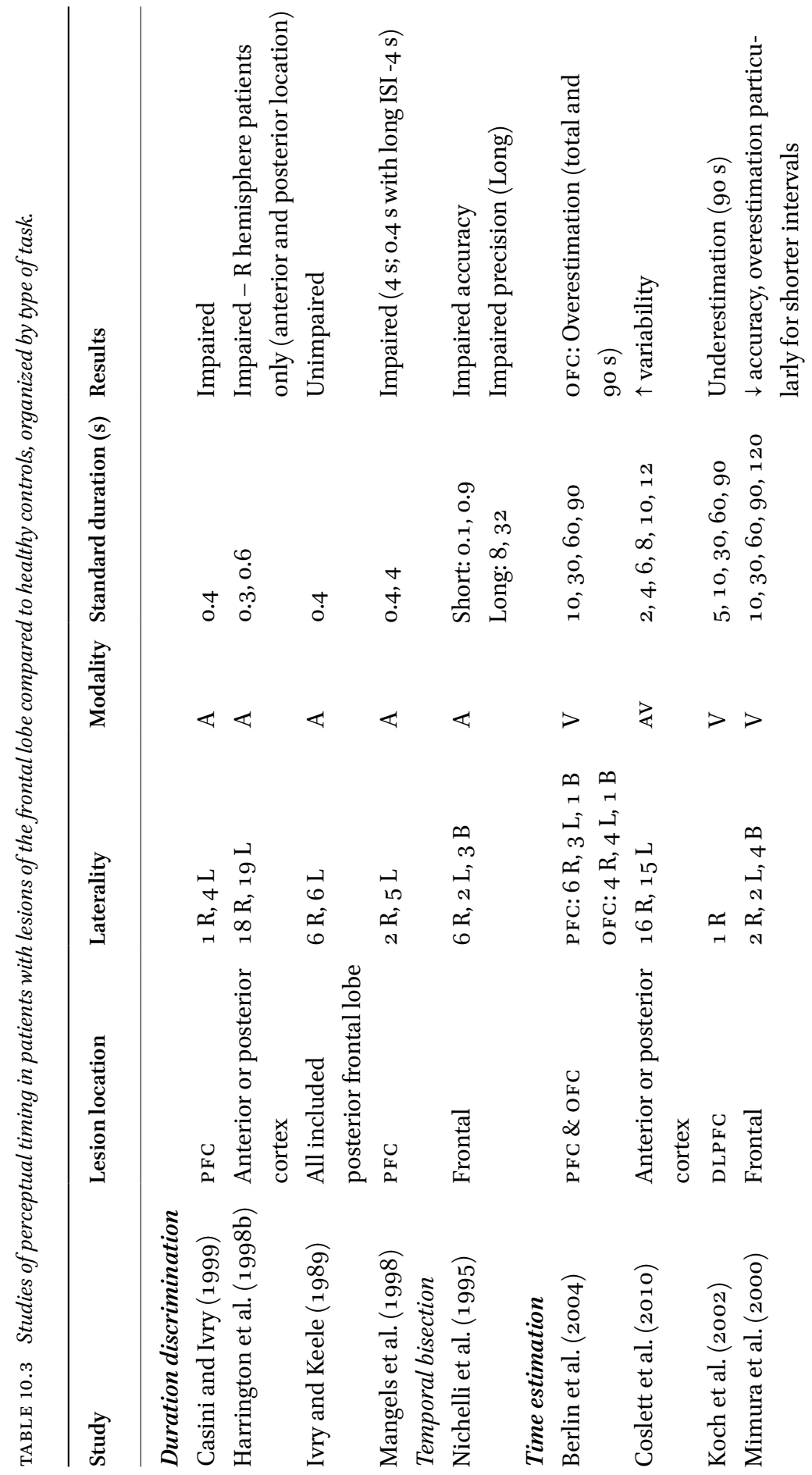




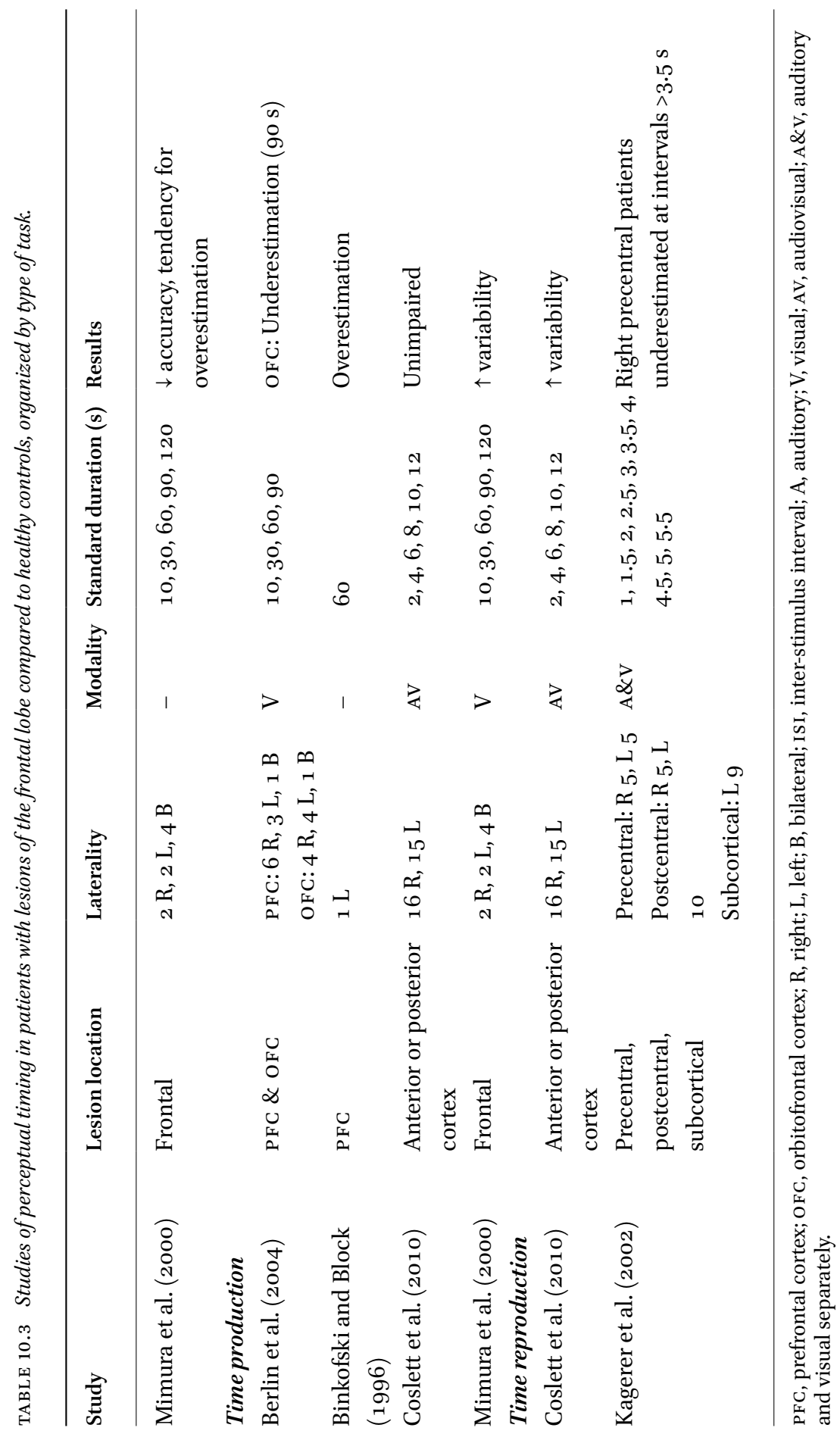


usual, with events (e.g., television programs) appearing to pass in 'accelerated motion'. Additionally, when driving, the velocity of his car seemed intolerably fast, with external objects rushing towards him at an excessive rate. Similarly, a patient with a lesion to the right DLPFC reported judging the durations of events in daily life as shorter than they actually were (Koch et al., 2002). This resulted in disruption to daily life, where the patient would leave work earlier than the scheduled time, convinced that the working day must have elapsed. However, data from the experimental measures of perceptual timing in both case reports presents apparently contradictory findings. Binkofski and Block's patient showed gross overproduction when asked to indicate to the examiner when 60 s had elapsed. If his subjective experience of accelerated time was due to a speeded internal clock (i.e., a clock with a pacemaker running too quickly) then he would have demonstrated underproduction. Similarly, Koch et al.'s patient showed underestimation when asked to verbally label the length of a go s period of time. A speeded clock would lead to overestimation, as more clock 'ticks' would accumulate in the designated interval. Further, performance for Koch et al.'s patient did not differ from control participants for intervals below 90 s. Koch and colleague's paradigm included reading aloud random numbers paced at a variable rate, which although inhibits counting is likely to have been differentially challenging for a patient with a PFC lesion. In summary, both studies demonstrate patients with PFC lesions who show remarkable and intrusive perceptual timing distortions. However, a straightforward clock explanation (i.e., a clock running too fast) does not fit with the pattern of experimental data. Related to this, Mimura et al. (2000) reported individual differences in the tendency to show either slowed or speeded responding in eight patients with frontal lesions tested on measures of time reproduction and time production. Thus, evidence for a simple speeded clock explanation in patients with frontal lesions is unlikely, mirroring the limited evidence for a slowed clock in PD. Of course, given the extent of the frontal cortex, it could be argued that clock speed is moderated by a specific region of the frontal cortex. Meta-analysis across patient studies, and neuroimaging and transcranial magnetic stimulation (TMS) in healthy populations could all be used to address this possibility.

Further investigation of the role of the PFC in timing was conducted by Casini and Ivry (1999), who tested duration discrimination (40oms standard) and frequency discrimination in patients with lesions to the $\mathrm{PFC}$, specifically the DLPFC. Compared to the results for the two tasks performed separately, the patients with DLPFC lesions showed impaired performance when the tasks were performed together. In contrast, the dual task manipulation only compromised duration discrimination for patients with cerebellar lesions. It was 
suggested that the timing deficits observed in patients with PFC lesions, unlike those with cerebellar lesions, can be explained by general deficits such as attention. Mangels et al. (1998) found that patients with lesions to the right or left PFC were impaired on a duration discrimination task when the intervals were long (4000 compared to 400ms) or the interval between the two durations was long (4000 compared to 1000ms). This suggests that it was the monitoring demands, specifically the executive control of working memory, rather than the temporal demands that drove the deficit. The patients with PFC lesions were also less able to spontaneously use regularly paced auditory tones to help them time the 40ooms interval, although they could benefit when given explicit instructions on how to utilize the markers. Again, this suggests that cognitive difficulties contributed to the poor timing performance. Harrington et al. (1998b) controlled for general cognitive deficits by excluding participants who were impaired at frequency discrimination. Of the remaining participants, duration discrimination was only impaired in patients with lesions to the right hemisphere (including the DLPFC and parietal cortex, particularly the supramarginal gyrus). The timing performance of the patients with right hemisphere lesions correlated with a measure of attention switching and the authors proposed a right prefrontal-inferior parietal network that influences temporal processing through its role in working memory and attention. In a final investigation of duration discrimination in patients with frontal lesions, Ivry and Keele (1989) found no evidence of impairment. Therefore, duration discrimination tasks tend toward showing a deficit in patients with frontal lesions but with the pattern of deficits consistent with non-specific cognitive processes driving the impairment.

A cognitive explanation was also the conclusion arrived at by Mimura et al. (2000). For their frontal patients, poor performance on the Wisconsin Card Sorting Task, a measure of executive functioning, correlated with increased verbal overestimation of units of time. Conversely, poor performance on a measure of delayed memory correlated with increased underestimation. Consequently, Mimura et al. argued that working memory deficits drove the atypical perceptual timing profile in patients with frontal lesions. However, it is also important to note that Coslett et al. (2009) did not find any correlation between performance on three perceptual timing tasks and the extent of damage in the lateral frontal lobe. In contrast, there were correlations between the extent of damage in the parietal lobe and performance on the time reproduction task.

Using an assessment of time reproduction, Kagerer et al. (2002) replicated Harrington et al.s (1998b) finding of compromised timing in patients with right hemisphere lesions only. Here, patients with lesions to the right and left 
hemispheres were unimpaired in the time reproduction of intervals $<3000 \mathrm{~ms}$ but patients with lesions to right precentral regions showed difficulty with intervals $>3500$ ms. Further, studies using repetitive TMS to create a 'virtual lesion' in healthy participants, report that disruption to the right DLPFC impairs temporal processing (e.g., Jones et al., 2004). However, the right hemisphere was not implicated in Coslett et al.'s (2009) study on perceptual timing in 31 patients with lesions across anterior and posterior cortex.

The severity of the temporal deficits seen in patients with frontal lesions is worth commenting on. The overestimation of the patients with OFC lesions in Berlin et al. (2004) was on average $20 \mathrm{~s}$ for a $90 \mathrm{~s}$ interval, although the large standard error bars suggest a considerable spread of responses. Mimura et al. (2000) reported at least two patients with remarkable overestimation, estimating $90 \mathrm{~s}$ as being greater than $150 \mathrm{~s}$. In contrast, another two patients estimated the same interval as being less than $50 \mathrm{~s}$. However, the task included a dual task component, reading aloud random numbers, which may have distorted the estimations. When the participants counted the intervals at the self-paced rate of $1 \mathrm{~s}$, performance was much improved, although one exceptionally poor performer remained. The patient assessed by Binkofski and Block (1996) produced average time production values of $286 \mathrm{~s}$, when he was asked to produce $6 \mathrm{o} \mathrm{s}$; while Koch et al.'s (2002) patient underestimated a $90 \mathrm{~s}$ interval as $40 \mathrm{~s}$. Deviations of this extent are not seen in patients with PD. For example, the PD patients described by Lange et al. (1995) estimated $60 \mathrm{~s}$ intervals as lasting about $40 \mathrm{~s}$ and produced time intervals of around $70 \mathrm{~s}$ when asked to produce $60 \mathrm{~s}$ intervals. These quantitative differences, as well as the tendency toward speeded vs. slowed responding, indicates that comparable pathology cannot be driving the deficits in patients with frontal lesions and those with PD. The most parsimonious explanation for the profile across patient groups is that aberrant cognitive processes are driving the extreme performance in the frontal patients, and that this cognitive disruption is less evident in the PD group and in patients with lesions to the basal ganglia.

\subsection{Motor Timing}

The classic S-C task has not been used very often to test patients with frontal lesions. Ivry and Keele (1989) assessed 12 patients with lesions that included damage to the posterior frontal lobe. The patients showed increased variability compared to controls during the continuation phase on a version of the task that required tapping every 550ms. Analysis using the Wing and Kristofferson (1973a,b) model attributed the variance to disruption in motor execution, rather than core clock processes. A larger study assessed 43 patients with a range of frontal lesions using a longer inter-tap interval of $1.5 \mathrm{~s}$ (Picton et al., 
2006). Increased variability on both phases of the task was found in patients with lesions to the right lateral frontal cortex, including some with lesions of the basal ganglia. In addition, patients with lesions to the superior medial frontal cortex showed competent performance in early trials but their variability became abnormally high as the task progressed. In contrast to Ivry and Keele, analysis using the Wing and Kristofferson model implicated clock processes in both types of dysfunction. More detailed investigation of the lesion locations led Picton et al. (2006) to attribute the role of the right lateral frontal cortex in monitoring time. Any temporal task naturally demands monitoring and the S-C task necessitates monitoring the clock, i.e., a counting of clock ticks, and also monitoring that the executed responses are accurate. Disruption to monitoring would lead to increased variability. This interpretation is compatible with the finding that the same patients were unable to effectively monitor foreperiods in a simple reaction time task (Stuss et al., 2005). In contrast, the deteriorating performance of the patients with lesions of the superior medial frontal cortex was related to the role of this region in sustaining performance and counteracting distraction.

Using a different type of task, Halsband et al. (1993) assessed patients with lesions to the primary motor cortex, premotor cortex or supplementary motor area (SMA) on a range of tasks that assessed rhythm reproduction from memory. These tasks used more complex rhythms than the simple isochronous S-C task. Lesions to both the premotor cortex and the SMA impacted upon rhythm reproduction, despite unimpaired performance on measures of rhythm discrimination and manual dexterity. The two patients with SMA lesions were assessed on their ability to tap in time with a complex rhythm and then in the absence of the pacing stimulus; thus the task was similar to the classic S-C task. Here, patients were only impaired when the pacing stimulus was absent. This suggested that it is the internal generation of remembered rhythms that is challenging, rather than timing with the external sensory guidance provided by cues. Notably, damage to the left hemisphere had more impact on performance than damage to the right hemisphere, with the effects being seen in both hands. The pattern of deficits suggests that these frontal premotor regions are necessary for the temporal aspects of motor programming to be successfully employed.

\subsection{Summary}

Localization of the key regions of the frontal cortex involved in temporal processing is limited in patient studies as lesion sites are varied. For example, the area of the frontal cortex that was most commonly compromised in Mangels et al.'s (1998) study was the DLPFC, but the lesions often extended to more ventral 
regions. Another limitation of this type of research is the plastic, compensatory changes that can occur following a lesion. The recruitment of alternative regions to enable performance on a task may mask or 'muddy' the results. Some studies have assessed patients with chronic lesions that are years old (e.g., 9-18 years in Mangels et al., 1998), whereas others have used patients with a more recent history (e.g., Coslett et al., 2009).

However, assessing across the perceptual and motor tasks it is clear that dysfunctional temporal processing is characteristic of patients with frontal lesions. Particularly, data are consistently interpreted as reflecting the impact of disrupted cognition, including working memory and monitoring, attention, and inhibition on performance.

Surveying the data, it is undisputable that patients with PD or lesions to the frontal cortex present with motor and perceptual timing deficits. The picture for patients with basal ganglia lesions is less clear, primarily because this patient group is understudied. However, the data suggest that when both the right and left basal ganglia are compromised motor timing deficits will emerge.

Seconds-range timing has been considered more cognitive (e.g., Lewis and Miall, 2003) and relying on the mesocortial pathway, while millisecond-range timing is argued to be the preserve of the nigrostriatal pathway (Rammsayer and Classen, 1997; Wiener et al., 2011). However, it is important to note that as PD progresses the extent of dysfunction extends beyond the frontostriatal motor loop. This includes connections from the ventral tegmental area in the midbrain to the frontal cortex (i.e., the mesocortical pathway). Recently, there has been discussion that executive dysfunction may influence, if not drive, the temporal processing deficits seen in PD (Parker et al., 2013). There is suggestion that perceptual timing requires the same resources, including those used in inhibition, updating, and shifting, that are employed during general executive processing (Brown et al., 2013). Therefore, damage to prefrontal regions that are engaged in executive functions should be sufficient to cause temporal disruption in PD. However, the temporal profile of patients with purely frontal damage is distinct from patients with PD. Most notably, temporal deficits, particularly perceptual, are more extreme in patients with frontal lesions. Further, although dopaminergic medication can ameliorate the motor symptoms of PD and predominantly has a positive effect on temporal processing, it does not consistently have a positive effect on executive performance (e.g., PascualSedano et al., 2008) and can even worsen performance (e.g., Cools and 
D'Esposito, 2011). Given the evidence of motor timing difficulties in patients with bilateral lesions to the basal ganglia (Coslett et al., 2010), it can be proposed that a core timing dysfunction in PD is exacerbated as the disease progresses and further frontal regions become compromised.

Merchant et al. (2013) have discussed how degeneracy offers a contributing explanation for why the profile of timing performance for a particular patient group can vary, both within and across studies. Degeneracy refers to the capacity of multiple neural systems to sustain the same behavioral function, such that damage to one neural system can be compensated by pre-existing neural systems that can support the behavior. Degeneracy can be observed in functional imaging studies when patients with PD recruit the cerebellum in the absence of adequate fronto-striatal activation and show behaviorally preserved performance (e.g., Jahanshahi et al., 2010). Further, it would explain why capturing temporal processing difficulties in patients with discrete lesions to the basal ganglia can prove elusive, particularly in cases where the lesion is unilateral and redundancy (i.e., the presence of identical mechanisms in both hemispheres) is also a factor. That a relatively intact frontal cortex, certainly in early stage PD, is not enough to compensate for temporal dysfunction suggests that the basal ganglia have a core role in interval timing.

The consideration of the contributory roles of the basal ganglia and associated cortical regions is sympathetic to current conceptualization of interval timing as being driven by both a core timing network and by areas that are activated in a context-dependent fashion (e.g., dependent on modality or cognitive load), rather than a single locus (see Merchant et al., 2013). The core timing network is proposed to engage cortical, thalamic, and basal ganglia structures, particularly the striatum and SMA (Merchant et al., 2013). This conceptualization fits well with the profile of timing deficits across the three patient groups explored in this chapter, although clearly patients with damage to the basal ganglia remain significantly understudied. A clear direction for future work would be to compare the three patient groups directly, assessing both cognitive and temporal performance, to enable detailed profiling of performance.

\section{References}

Alexander, G.E., M.R. DeLong, and P.L. Strick. 1986. "Parallel Organization of Functionally Segregated Circuits Linking Basal Ganglia and Cortex" Annu Rev Neurosci 9: 357-81.

Aparicio, P., J. Diedrichsen, and R.B. Ivry. 2005. "Effects of Focal Basal Ganglia Lesions on Timing and Force Control" Brain Cogn 58(1): 62-74. 
Berlin, H.A., E.T. Rolls, and U. Kischka. 2004. "Impulsivity, Time Perception, Emotion and Reinforcement Sensitivity in Patients with Orbitofrontal Cortex Lesions" Brain 127(5): 1108-26.

Binkofski, F., and R.A. Block. 1996. "Accelerated Time Experience after Left Frontal Cortex Lesion" Neurocase 2: 485-93.

Brown, R.G., and C.D. Marsden. 1991. "Dual Task Performance and Processing Resources in Normal Subjects and Patients with Parkinson's Disease" Brain 114(1): 215-31.

Brown, S.W., S.A. Collier, and J.C. Night. 2013. "Timing and Executive Resources: DualTask Interference Patterns between Temporal Production and Shifting, Updating, and Inhibition Tasks" J Exp Psychol Hum Percept Perform 39(4): 947-63.

Casini, L., and R.B. Ivry. 1999. "Effects of Divided Attention on Temporal Processing in Patients with Lesions of the Cerebellum or Frontal Lobe" Neuropsychology 13(1): $10-21$.

Cerasa, A. et al. 2006. "Functional Changes in the Activity of Cerebellum and Frontostriatal Regions during Externally and Internally Timed Movement in Parkinson's Disease" Brain Res Bull 71(1-3): 259-69.

Chaudhuri, K.R., and A.H. Schapira. 2009. "Non-Motor Symptoms of Parkinson's Disease: Dopaminergic Pathophysiology and Treatment" Lancet Neurol 8(5): 464-74. Claassen, D.O. et al. 2013. "Deciphering the Impact of Cerebellar and Basal Ganglia Dysfunction in Accuracy and Variability of Motor Timing" Neuropsychologia 51: 267-74.

Cools, R., and M. D’Esposito. 2011. "Inverted-U-Shaped Dopamine Actions on Human Working Memory and Cognitive Control" Biol Psychiatry 69(12): e113-25.

Coslett, H.B. et al. 2009. "Cognitive Timing: Neuropsychology and Anatomic Basis" Brain Res 1254: 38-48.

Coslett, H.B., M. Wiener, and A. Chatterjee. 2010. "Dissociable Neural Systems for Timing: Evidence from Subjects with Basal Ganglia Lesions" PLoS One 5(4): e10324.

Coull, J., and A. Nobre. 2008. "Dissociating Explicit Timing from Temporal Expectation with Fmri” Curr Opin Neurobiol 18(2): 137-44.

D'Esposito, M. 2007. "From Cognitive to Neural Models of Working Memory." Philos Trans R Soc Lond B Biol Sci 362(1481): 761-72.

Dirnberger, G., and M. Jahanshahi. 2013. “Executive Dysfunction in Parkinson's Disease: A Review [In eng]" J Neuropsychol 7(2): 193-224.

Duchek, J.M. et al. 1994. "Component Analysis of a Rhythmic Finger Tapping Task in Individuals with Senile Dementia of the Alxheimer Type and in Individuals with Parkinson's Disease" Neuropsychology 8(2): 218-26.

Elsinger, C.L. et al. 2003. "Neural Basis for Impaired Time Reproduction in Parkinson's Disease: An Fmri Study” J Int Neuropsychol Soc 9(7): 1088-98.

Gibbon, J. 1977. “Scalar Expectancy Theory and Weber's Law in Animal Timing” Psychol $\operatorname{Rev} 84:$ 279-325.

Gibbon, J. et al. 1984. "Scalar Timing in Memory" Ann N Y Acad Sci 423: 52-77. 
Grahn, J.A., and M. Brett. 2009. "Impairment of Beat-Based Rhythm Discrimination in Parkinson's Disease" Cortex 45(1):54-61.

Guehl, D. et al. 2008. "Auditory Temporal Processing in Parkinson's Disease" Neuropsychologia 46(9): 2326-35.

Halsband, U. et al. 1993. "The Role of Premotor Cortex and the Supplementary Motor Area in the Temporal Control of Movement in Man" Brain 116(1): 243-66.

Harrington, D.L., K.Y. Haaland, and N. Hermanowicz. 1998a. "Temporal Processing in the Basal Ganglia" Neuropsychology 12(1): 3-12.

Harrington, D.L., K.Y. Haaland, and R.T. Knight. 1998b. "Cortical Networks Underlying Mechanisms of Time Perception" JNeurosci 18(3): 1085-95.

Hellström, A. et al. 1997. "Tone Duration Discrimination in Parkinson's Disease" Neuropsychologia 35(5): 737-40.

Hinton, S.C., Harrington, D.L., Binder, J.R., Durgerian, S., and Rao, S.M. 2004. "Neural Systems Supporting Timing and Chronometric Counting: an fMRI Study" Cog Brain Res, 21(2): 183-92.

Ivry, R.B., and S.W. Keele. 1989. "Timing Functions of the Cerebellum" J Cogn Neurosci $1(2): 136-52$.

Jahanshahi, M. et al. 2006. "The Substantia Nigra Pars Compacta and Temporal Processing” J Neurosci 26(47): 12266-73.

Jahanshahi, M. et al. 2010. "Dopaminergic Modulation of Striato-Frontal Connectivity during Motor Timing in Parkinson's Disease" Brain 133(3): 727-45.

Jones, C.R.G. and Jahanshahi, M. 2014. "Contributions of the Basal Ganglia to Temporal Processing: Evidence from Parkinson's Disease" Timing \& Time Perception, 2(1): 87-127.

Jones, C.R. et al. 2004. "The Right Dorsolateral Prefrontal Cortex is Essential in Time Reproduction: An Investigation with Repetitive Transcranial Magnetic Stimulation" Exp Brain Res 158(3): 366-72.

Jones, C.R. et al. 2008. "Basal Ganglia, Dopamine and Temporal Processing: Performance on Three Timing Tasks On and Off Medication in Parkinson's Disease" Brain Cogn 68(1):30-41.

Jones, C.R.G. et al. 2011. "Modeling Accuracy and Variability of Motor Timing in Treated and Untreated Parkinson's Disease and Healthy Controls" Front Integr Neurosci $5(81)$.

Joundi, R.A. et al. 2012. "High-Frequency Stimulation of the Subthalamic Nucleus Selectively Decreases Central Variance of Rhythmic Finger Tapping in Parkinson's Disease" [In eng] Neuropsychologia 50(10): 2460-6.

Kagerer, F.A. et al. 2002. "Cortical Involvement in Temporal Reproduction: Evidence for Differential Roles of the Hemispheres" Neuropsychologia 40(3): 357-66.

Katsuki, F., and C. Constantinidis. 2012. "Unique and Shared Roles of the Posterior Parietal and Dorsolateral Prefrontal Cortex in Cognitive Functions" Front Integr Neurosci 6: 17 .

Keele, S.W. et al. 1985. "Do Perception and Motor Production Share Common Timing Mechanisms: A Correctional Analysis" Acta Psychol 6o(2-3): 173-91. 
Kehagia, A.A., R.A. Barker, and T.W. Robbins. 2013. “Cognitive Impairment in Parkinson's Disease: The Dual Syndrome Hypothesis" Neurodegener Dis 11(2): 79-92.

Koch, G. et al. 2002. "Selective Deficit of Time Perception in a Patient with Right Prefrontal Cortex Lesion" Neurology 59(10): 1658-9.

Koch, G. et al. 2004. "Subthalamic Deep Brain Stimulation Improves Time Perception in Parkinson's Disease" Neuroreport 15(6): 1071-3.

Koch, G. et al. 2005. "Memory for Time Intervals is Impaired in Left Hemi-Parkinson Patients" Neuropsychologia 43(8): 1163-7.

Koch, G. et al. 2008. "Impaired Reproduction of Second but Not Millisecond Time Intervals in Parkinson's Disease" Neuropsychologia 46(5): 1305-13.

Lange, K.W. et al. 1995. "Subjective Time Estimation in Parkinson's Disease" J Neural Transm Suppl 46: 433-8.

Lewis, P.A., and R.C. Miall. 2003. "Distinct Systems for Automatic and Cognitively Controlled Time Measurement: Evidence from Neuroimaging" Curr Opin Neurobiol 13(2): 250-5.

Malapani, C. et al. 1998. "Coupled Temporal Memories in Parkinson's Disease: A Dopamine-Related Dysfunction" J Cogn Neurosci 10(3): 316-31.

Malapani, C., B. Deweer, and J. Gibbon. 2002. "Separating Storage from Retrieval Dysfunction of Temporal Memory in Parkinson's Disease" JCogn Neurosci 14(2): 311-22.

Mangels, J.A., Ivry, R.B., and Shimizu, N. 1998. "Dissociable Contributions of the Prefrontal and Neocerebellar Cortex to Time Perception" Cogn Brain Res 7: 15-39.

Matell, M.S., and W.H. Meck. 2000. "Neuropsychological Mechanisms of Interval Timing Behavior" Bioessays 22(1): 94-103.

Matell, M.S., and W.H. Meck. 2004. "Cortico-Striatal Circuits and Interval Timing: Coincidence Detection of Oscillatory Processes" Brain Res Cogn Brain Res 21(2):139-70.

Merchant, H. et al. 2008. "Interval Timing and Parkinson's Disease: Heterogeneity in Temporal Performance" Exp Brain Res 184(2): 233-48.

Merchant, H., D.L. Harrington, and W.H. Meck. 2013. "Neural Basis of the Perception and Estimation of Time” Annu Rev Neurosci 36: 313-36.

Mimura, M., M. Kinsbourne, and M. O'Connor. 2000. "Time Estimation by Patients with Frontal Lesions and by Korsakoff Amnesics" J Int Neuropsychol Soc 6(5): 517-28.

Nichelli, P. et al. 1995. "Duration Processing after Frontal Lobe Lesions" Ann NY Acad Sci 769: 183-9o.

O’Boyle, D.J., J.S. Freeman, and F.W. Cody. 1996. “The Accuracy and Precision of Timing of Self-Paced, Repetitive Movements in Subjects with Parkinson's Disease" Brain 119(1): 51-70.

Parker, K.L. et al. 2013. "Executive Dysfunction in Parkinson's Disease and Timing Deficits" Front Integr Neurosci 7: 75.

Pascual-Sedano, B. et al. 2008. "Levodopa and Executive Performance in Parkinson's Disease: A Randomized Study" J Int Neuropsychol Soc 14(5): 832-41. 
Pastor, M.A. et al. 1992a. “Time Estimation and Reproduction Is Abnormal in Parkinson's

Disease" Brain 115(1): 211-25.

Pastor, M.A. et al. 1992b. "Performance of Repetitive Wrist Movements in Parkinson's Disease" Brain 115(3): 875-91.

Perbal, S. et al. 2005. "Effects of Internal Clock and Memory Disorders on Duration Reproductions and Duration Productions in Patients with Parkinson's Disease" Brain Cogn 58(1): 35-48.

Picton, T.W. et al. 2006. "Keeping Time: Effects of Focal Frontal Lesions" Neuropsychologia 44(7): 1195-209.

Rammsayer, T., and W. Classen. 1997. “Impaired Temporal Discrimination in Parkinson's Disease: Temporal Processing of Brief Durations as an Indicator of Degeneration of Dopaminergic Neurons in the Basal Ganglia" Int J Neurosci 91(1-2): 45-55.

Riesen, J.M., and A. Schnider. 2001. “Time Estimation in Parkinson's Disease: Normal Long Duration Estimation Despite Impaired Short Duration Discrimination" $J$ Neurol 248(1): 27-35.

Schwartze, M. et al. 2011. "The Impact of Basal Ganglia Lesions on Sensorimotor Synchronization, Spontaneous Motor Tempo, and the Detection of Tempo Changes" Behav Brain Res 216(2): 685-91.

Smith, J.G. et al. 2007. "The Effect of Parkinson's Disease on Time Estimation as a Function of Stimulus Duration Range and Modality" Brain Cogn 64(2): 130-43.

Spencer, R.M., and R.B. Ivry. 2005. “Comparison of Patients with Parkinson's Disease or Cerebellar Lesions in the Production of Periodic Movements Involving Event-Based or Emergent Timing" Brain Cogn 58(1): 84-93.

Stuss, D.T. et al. 2005. "Multiple Frontal Systems Controlling Response Speed" Neuropsychologia 43(3): 396-417.

Torregrossa, M.M., J.J. Quinn, and J.R. Taylor. 2008. "Impulsivity, Compulsivity, and Habit: The Role of Orbitofrontal Cortex Revisited" Biol Psychiatry 63(3): 253-5.

Torta, D.M. et al. 2010. "Dissociation between Time Reproduction of Actions and of Intervals in Patients with Parkinson's Disease" J Neurol 257(8): 1356-61.

Wearden, J.H. et al. 2008. "Stimulus Timing by People with Parkinson's Disease" Brain Cogn $67(3): 264-79$.

Wiener, M., F.W. Lohoff, and H.B. Coslett. 2011. "Double Dissociation of Dopamine Genes and Timing in Humans" J Cogn Neurosci, 23(10): 2811-21.

Wild-Wall, N. et al. 2008. "Time Estimation in Healthy Ageing and Neurodegenerative Basal Ganglia Disorders" Neurosci Lett 442: 34-8.

Wing, A.M., and Kristofferson., A.B. 1973a. "Response Delays and Timing of Discrete Motor Responses" Percept Psychophys 14: 5-12.

Wing, A.M., and Kristofferson., A.B. 1973 "Timing of Interresponse Intervals" Percept Psychophys 13: 455-6o.

Wojtecki, L. et al. 2011. "Modulation of Human Time Processing by Subthalamic Deep Brain Stimulation" PLoS One 6(9): 12. 